\title{
A Trial for Accuracy Improvement on Fibrinogen Assay
}

\author{
Noriyuki TATSUMI ${ }^{1}$; Masaharu YoKOTA ${ }^{2}$; Hiroshi Kondo ${ }^{3}$; and Masahiro OKUDA ${ }^{4}$ \\ ${ }^{1}$ International Buddhist University, ${ }^{2}$ Health and Hygiene Central of Sakai City. ${ }^{3}$ Osaka College of Health Sciences, and ${ }^{4}$ International Reagent Co.
}

\begin{abstract}
The performance of coagulation testing including fibrinogen assay is determined by a combination of reagents and instruments. Almost all coagulation tests are automated using commercially-produced reagents and automated analyzer based on the clotting principle, although the diversity of the combination results in high variation of test results.

We analyzed our results of external quality surveillance in past 10 years, high variation of the fibrinogen assay was observed. Thus, we tried to produce a reference material for the assay and studied if the use of the material could help to improve the high variation.

However, the material was not be able to improve the variation. We, therefore, concluded that current high variation of fibrinogen assay was not caused by not only diversity of reagents but also that of coagulation test analyzers.

Key Words Fibrinogen, External Quality assurance, Accuracy, Clot Assay
\end{abstract}

\section{INTRODUCTION}

Following the changes in dietary habit, risks of cardiovascular disorders have been increasing, such as deep vein thrombosis and coronary artery sclerosis. To monitor the risks and treatment for the disorders, the needs of three main coagulation tests has been increasing; Prothrombin Time (PT), Activated Partial Thromboplastin Time (APTT) and Fibrinogen concentration (Fbg) assays. The 3 kinds of the tests are basically measured in the routine hematology laboratory using an automated coagulometer. As all of 3 assays are based on the fibrinogen-fibrin conversion principle, purity and biological activity of the fibrinogen in the test material determine the function of those tests. Calibration materials are used routinely to assign determined values. Thus, a good calibration material is required for its value assignment.

Previously we developed a reference material candidate for an accurate fibrinogen assay. ${ }^{[1]}$ This paper describes a retrospective study on fibrinogen assays and an experimental study for evaluating efficacy of our reference material.

\footnotetext{
Received: May 10, 2004

${ }^{1}$ Address; International Buddhist University, 3-2-1, Gakuenmae, Habikino, Osaka, 583-8501 Japan. TEL: 81-729-56-3181

${ }^{2}$ Address; Health and Hygiene Central of Sakai City, Japan. TEL: 81-72-238-1848

${ }^{3}$ Address; Osaka College of Health Sciences, 3-2-49, Habikino, Habikino, Osaka, 583-8555 Japan. TEL: 81-729-50-2111
}

\section{MATERIALS AND METHODS}

\section{Retrospective studies on accuracy and precision of fib-} rinogen assay

Japan Medical Association has been conducting an External Quality Assessment Schema (EQAS-J) once every year for participants from whole country in Japan. We joined the EQAS as members of its committee and have been provided surveillance materials for 3 coagulation tests; PT, APTT and Fbg. ${ }^{[2]-[4] ~ R e s u l t s ~ o f ~ t h e ~ E Q A S ~ w e r e ~ e x h i b i t e d ~ i n ~} 2$ forms; Yuden's X-Y plot figures and box-and-hinge charts in each item.

\section{Evaluation of our reference material}

We called many of coagulation laboratories for joining our trial, and got 154 participants from whole country. Each laboratory determined Fbg concentration with their own test reagents and a instruments using two calibration materials; the one was 2 concentration manufacturer's-made reference materials that each laboratory kept, and the other was 2 concentrations of our reference materials that we offered. The trial was conducted in October 2003. A data set of the determined values was reported to us by e-mail and summarized for comparison and evaluation.

\section{RESULTS}

\section{Variation changes in fibrinogen assay in EQAS-J}

As shown in Fig. 1, the 2000 surveillance showed wider distribution on the Fbg assays when 2 concentration materials were studied, while better and concentrated distribution were shown in the 2003 surveillance. ${ }^{[4]}$ Some of dots were positioned out of circles in the former, and almost all dots were positioned near the distribution center in the latter. Before 2000, many reagents for the PT assay were employed their ISI (International Sensitivity Index) power over 2.0. The Surveillance Committee in Japan Medical Association called all reagent manufacturers for use of high sensitivity reagent with the power of below 1.7 of ISI for PT assay. At the same time our committee asked them to harmonize all Fbg levels. Since then, ISI values have been gradually lowered and Fbg concentration also was centralized among manufacturers.

Figure 2 shows year-by-year changes in fibrinogen assays, gradual improvement was able to observe in two evaluation parameters on the Fbg assay; inter-laboratory and intra-laboratory variations. Intra-reagent $\mathrm{CVs}$ (coefficient of variation) has been gradually decreased from 1995 to 2003, while intrareagent CVs has been kept almost the same level at about 7 to 

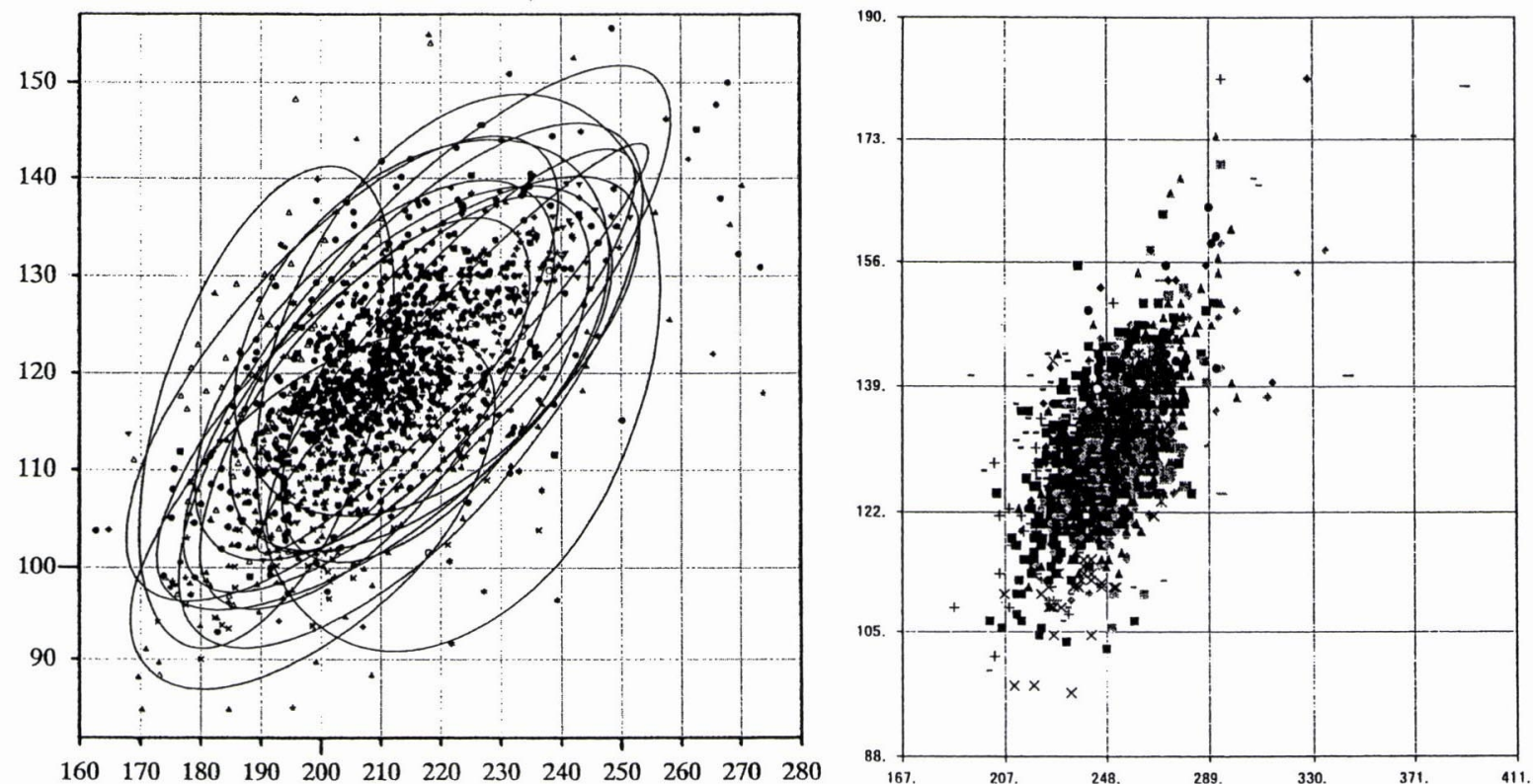

Fig. 1 Yuden plots (X-Y plotted charts) of EQAS-J results on fibrinogen assays. ${ }^{[3],[4]}$

Reported results of two concentration materials ( $\mathrm{X}$ axis and $\mathrm{Y}$ axis) distributed were plotted on a chart. Scale; Fbg concentration, $\mathrm{mg} / \mathrm{d} l$.

(Left) Surveillance results in 2000, $N=1,810$, each symbol shows a result of a manufacture's reagent group in 2000. (Right) (Surveillance results in 2003, $N=1,831$, each symbol shows a result of a manufacturer's reagent group in 2003.

Fibrinogen

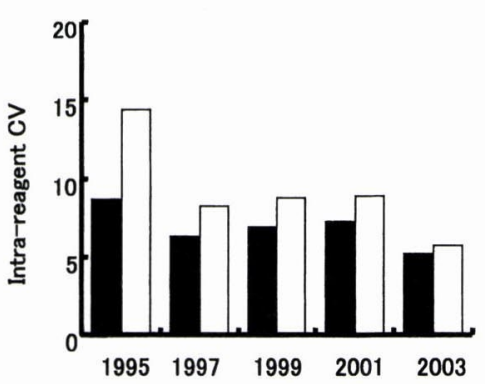

Fibrinogen

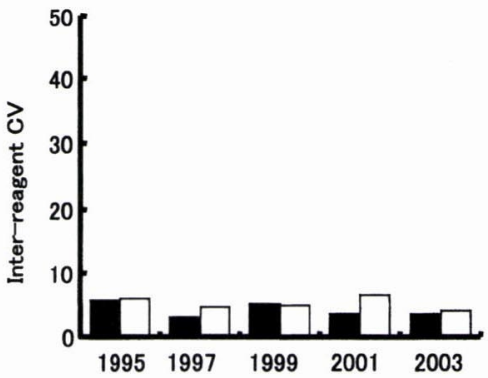

Fig. 2 Year-by-year changes in inter-reagent variations and intra-reagent variation from 1995 to 2003 in EQAS-J surveillance. Two materials (normal concentration one in a black column and low concentration one in white column) have been distributed. $\mathrm{X}$ axis; year. $\mathrm{Y}$ axis; $\mathrm{CV}$ in $\%$.
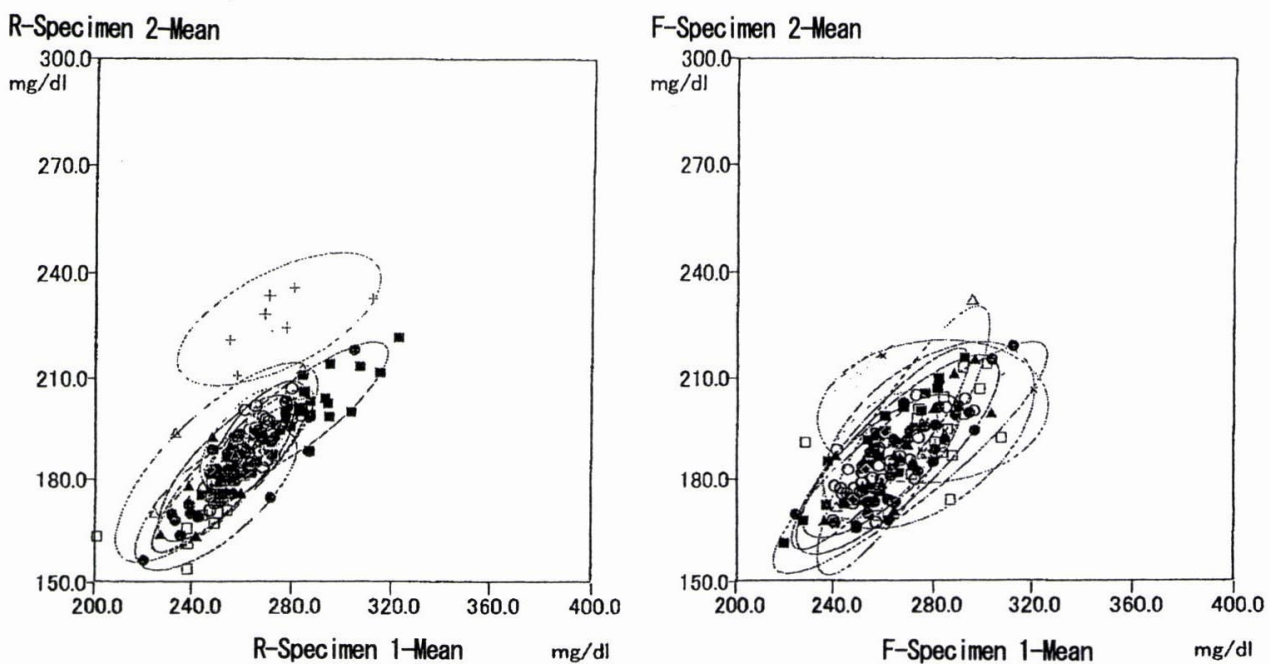

Fig. 3 Comparison of calibration efficacy study in 2 reagents; routine calibration reagents.

$\mathrm{R}$; routine calibration material (left figure). F; our fibrinogen reference material (right figure). Two materials were determined (X and $\mathrm{Y}$ axis each). Left; $N=154$. $r=0.7836$. Right; $N=164$. $r=0.7467$. Scale on $\mathrm{X}$ and $\mathrm{Y}$ axes; Fbg concentration, $\mathrm{mg} / \mathrm{d} l$. 
5\% during the period. And in 2003, inter-reagent CVs became less than $5 \%$, although the value might be not satisfactory one as a bioactivity assay on fibrinogen concentration, compared to the levels of chemistry tests. ${ }^{[5]}$

\section{Evaluation of our reference material for fibrinogen} assay

As the CVs for the Fbg assay were judged to be very high, ${ }^{[1]}$ we tried to distribute our Fbg reference material to taper the CV values among laboratories. Almost all laboratories have used an coagulation analyzer based on the clotting principle. We fractionated fresh human plasma to make purified plasma showing the same responsiveness on Fbg assay as the fresh human plasma behave. The plasma we made possessed high purity over $98 \%$ of fibrinogen. The plasma was distributed to our participants to use it as same as they handled the routine calibration material.

As Fig. 3 shows, the group using routine materials (left) formed a smaller cluster along the diagonal line than the size of the group using our material (right), when a cluster with a cross mark was excluded in the left figure. The results indi-

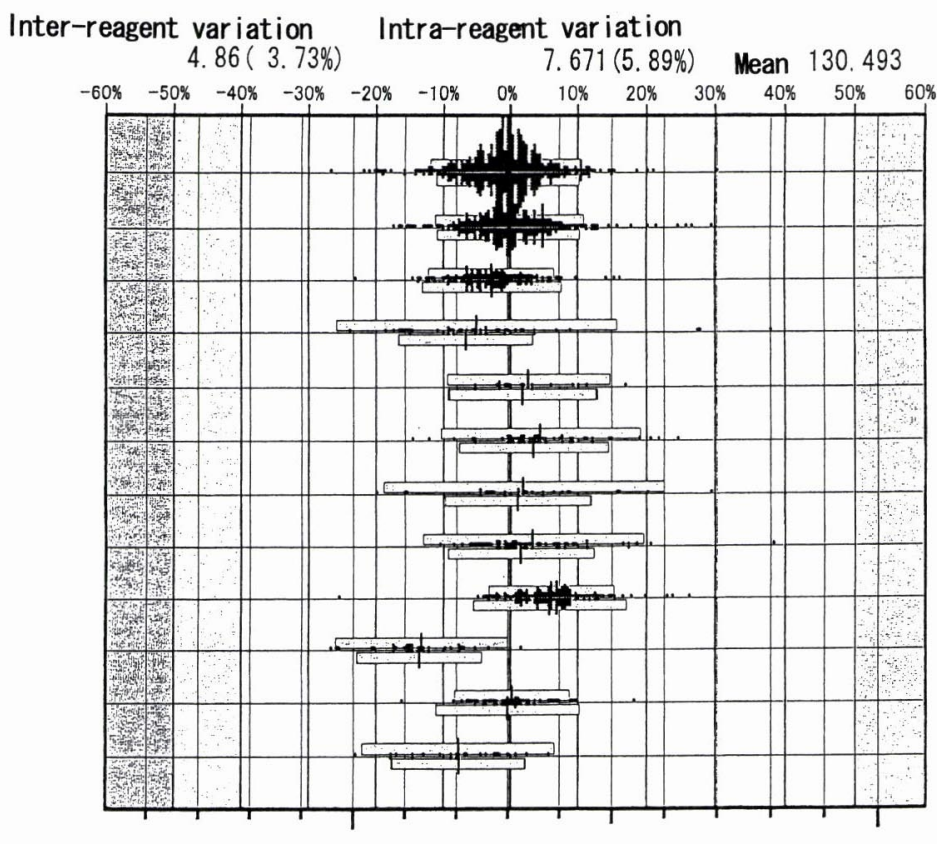

$\begin{array}{lllllllllllllll}60 & 70 & 80 & 90 & 100 & 110 & 120 & 130 & 140 & 150 & 160 & 170 & 180 & 190 & 200\end{array}$

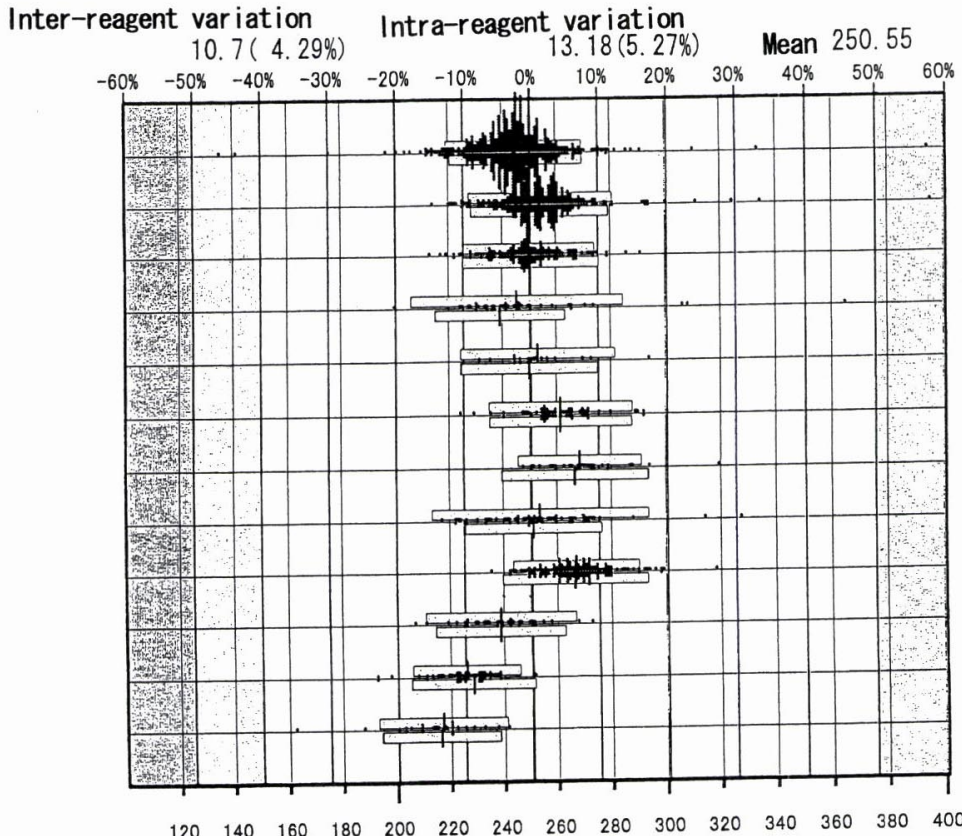

Fig. 4 Box and hinge charts in each reagent group in fibrinogen assays. ${ }^{[4]}$

The data was extracted from 2003 surveillance results and classified by reagent groups.

The participant mean is expressed as $0 \%$ shown on the $\mathrm{X}$ axis on the top of the figure, and as real mean concentration of $\mathrm{mg} / \mathrm{d} l$ shown under the figure. Each line shows one reagent group. Upper Square means a statistical analysis data by a common method on a line, and Lower Square means a specific analysis data designed by Ichihara's calculation formulas under the line. (From top to bottom) $N=624,400,184,43,18$, 58, 29, 63, 166, 37, 56, 24. (Upper) Low concentration material. (Lower) High concentration material. 

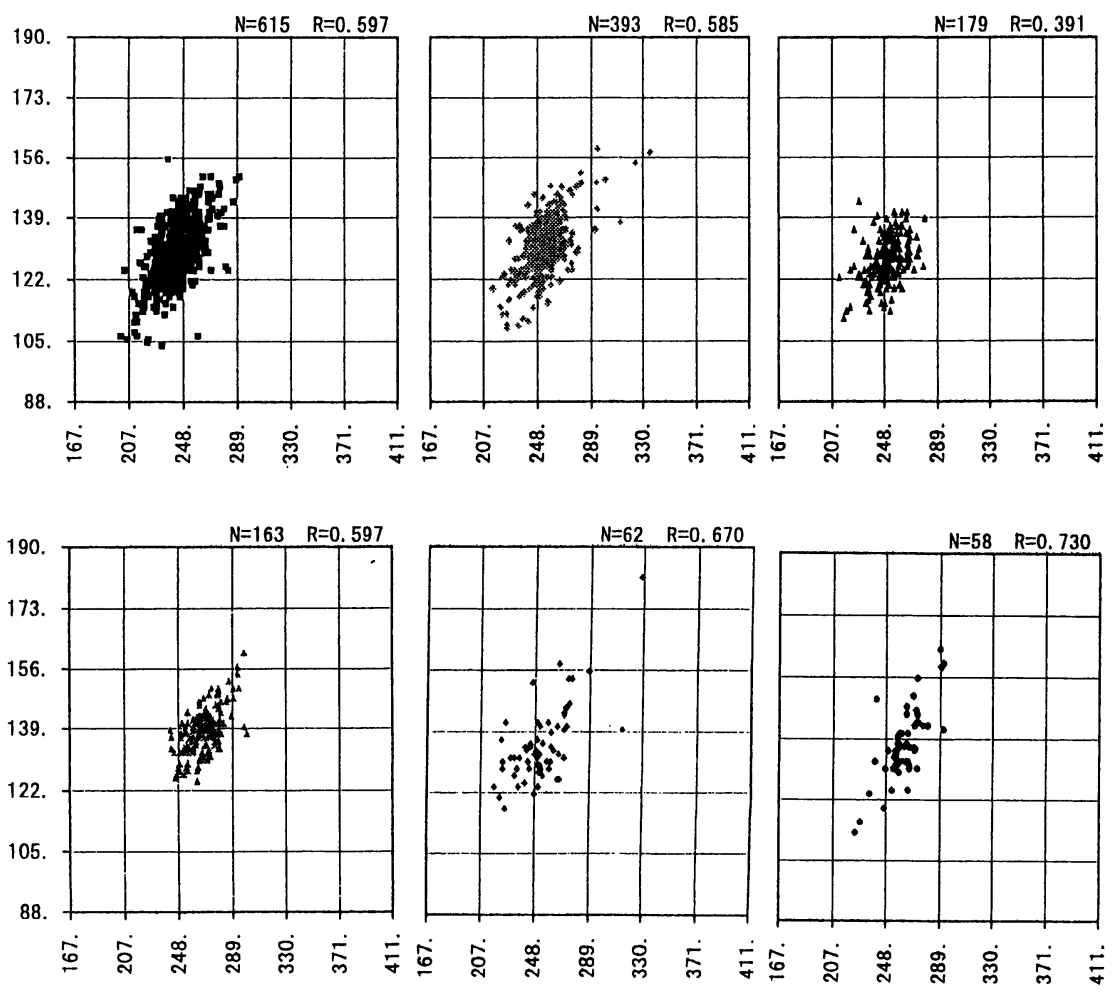

Fig. 5 Yuden plots (X-Y plotted charts) in 6 reagent groups. ${ }^{[4]}$

The data was extracted from 2003 surveillance results and classified by reagent groups. Characteristic patterns can be observed in each reagent group. Scale on X and Y axes.

cated us that the use of purified material was unable to help improvement of the high variation of Fbg values.

\section{DISCUSSION}

We have conducted nationwide external quality schemas for over 10 years providing our surveillance materials to all participants under the auspices of Japan Medical Association. ${ }^{[6]}$ The results have provided us much and useful information for production of better and safe materials for the surveillance in all filed of laboratory medicine. However, coagulation test materials were pain in the neck for us since high variation of determined values was unable to improve on tests of PT, APTT, and Fbg. In the context, a reference material for fibrinogen assay was developed by us to aim accuracy improvement of the determination. ${ }^{[1]}$

Our pilot study showed that our material was able to contribute to determined value unification of fibrinogen concentration assay. Thus, we thought that the use of our material would be useful to harmonize all fibrinogen assays throughout country, and we designed this study. Our material was used to be used for instrument calibration in each laboratory, and the material was distributed for aiming routine calibration use. However, the results this time were not so good since it failed to taper the $\mathrm{CV}$ value of the Fbg values. Thus we judged that the common use of our purified material was not helpful to harmonize.

Thereafter, we rechecked the Fbg concentration assay on reagent dependency, referring the past EQAS-J. As Figs. 4 and 5 shows, it was shown that determined values would have strong dependency on reagent specificity. The Japan Medical Association Report on recent clinical laboratory tests said that 3 major parameters (PT, APTT, Fbg) were determined now using automated analyzers in Japan, and that most of coagulation laboratories adopted instrument-specific reagents. As all of those tests were principally determined by fibrinogen-fibrin conversion reaction, accurate content must be known in each reagent, since the Fbg content determines the performance and precision of each analyzer. When we evaluate performance of 3 coagulation tests (OT, APTT, Fbg), we should consider analyzer's function on fitness between a reagent and an analyzer. In that sense, accurate measurement would be desired to perform high quality coagulation tests.

Importance of health check-up is now increasing. We cannot, however, evaluate heath conditions without coagulation test data, since arteriosclerosis-associated health risks and threat are now increasing. For monitoring the thrombosis risks, it would be needed to determine Fbg concentration as accurate as possible.

\section{REFERENCES}

[1] Okuda M: Quality Control Material for Plasma Fibrinogen Material for Plasma Fibrinogen Test Produced from Purified Human Fibirinogen. J Automated Methods Management in Chem 25: 79-85, 2003

[2] Tatsumi N: Hematological Tests. In The 31st Surveillance Report of External Quality Assurance Schema in 1997. 
pp84-106, Japan Medical Association, Tokyo, 1997

[3] Tatsumi N: Hematological Tests. In The 34 th Surveillance Report of External Quality Assurance Schema in 2000. pp107-139, Japan Medical Association, Tokyo, 2000

[4] Tatsumi N: Hematological Tests. In The 37 th Surveillance Report of External Quality Assurance Schema in 2003. pp117-134, Japan Medical Association, Tokyo, 2003
[5] Ichihara K: Statistical Analysis. In The 37 th Surveillance Report of External Quality Assurance Schema in 2003. pp135-195, Japan Medical Association, Tokyo, 2003

[6] Tatsumi N: Hematological Tests. In Medical Tests Quality Control; 30 Years History (ed: Kawai K), pp29-51, Japan Medical Association, Tokyo, 1998 